\title{
Hidrogéis Superabsorventes a Base de Acetato de Celulose Modificado por Dianidrido 3, 3', 4, 4' Benzofenona Tetracarboxílico (BTDA): Síntese, Caracterização e Estudos Físico-Químicos de Absorção
}

\author{
Vagner R. Botaro \\ UFSCar/Campus de Sorocaba
}

\author{
Cláudio G. Santos \\ DEQUI, UFOP
}

\section{Victor A. Oliveira \\ DEMET, UFOP}

\begin{abstract}
Resumo: Este trabalho descreve as sínteses de hidrogéis superabsorventes a base de acetato de celulose (AC), com grau de substituição 2.5, reticulado com dianidrido 3, 3', 4, 4' benzofenona tetracarboxílico (BTDA). As caracterizações das matérias primas foram realizadas por análises térmicas (TG/DTG) e espectroscopia na região do infravermelho (FTIR). O grau de substituição do acetato de celulose foi determinado por via úmida. A confirmação da reação de esterificação foi possível a partir da técnica de FTIR, espectroscopia UV-vis e análises térmicas (TG/DTG). A presença de poros no novo material foi analisada por microscopia eletrônica de varredura (MEV) e porosimetria (BET). A influência da concentração de dianidrido no meio reacional foi investigada, bem como a influência do aumento no grau de reticulação no comportamento térmico do material. Foram obtidas isotermas de absorção de água em diferentes temperaturas para os hidrogéis com diferentes graus de reticulação. Os coeficientes de difusão dos géis foram determinados, juntamente com a energia de ativação para o processo de inchamento usando a equação de Arrhenius. A entalpia de mistura do sistema gel-água foi determinada pela medida da quantidade máxima de água absorvida no equilíbrio a diferentes temperaturas, empregando a equação de Gibbs/ Helmholtz.
\end{abstract}

Palavras-chave: Hidrogéis, acetato de celulose, entalpia de mistura.

\section{Synthesis of Hydrogels of Cellulose Acetate (AC) Cross-Linked With 3,3',4,4' Benzophenonetetracarboxylic Dianhydride (BTDA): Characterization and Adsorption Physicochemical Study}

Abstract: This work describes the synthesis of hydrogels of cellulose acetate (AC), with a nominal degree of substitution $\mathrm{DS}=2.5$, cross-linked with 3,3',4,4' benzophenonetetracarboxylic dianhydride (BTDA). The raw materials were characterized by thermal analysis (TG/DTG) and by Fourier transform infrared spectroscopy (FTIR). DS of cellulose acetate was determined by titration with a known amount of standard $\mathrm{NaOH}$ solution. Hydrogels of BTDA were synthesized with $0.5,0.75$ and $1.0 \mathrm{~mol}$ of BTDA $/ \mathrm{mol}$ of AC. FTIR proved to be a suitable method to monitor the course of reactions and the progress of purification. UV-vis spectroscopy and analysis confirmed the esterification of the free hydroxyl groups. Surface modification of AC structure after the cross-linking reaction was analyzed by Scanning Electron Microscope (SEM) and density and porosity of the hydrogels were determined by BET. The influence of the concentration of dianhydride on the time necessary for formation of the gel was investigated. The influence from the increase in the degree of cross-linking on the thermal behavior of the material was also documented. Water absorption isotherms were obtained for hydrogels with different reticulating agents and reticulation degrees at different temperatures. The Arrhenius equation was used to determine the diffusion coefficient of the different hydrogels at distinct temperatures and the threshold energy for the swelling process. The enthalpy of mixture was determined through the measurement of the maximum quantity of water absorbed at equilibrium at different temperatures, with the Gibbs/Helmholtz equation.

Keywords: Hydrogels, cellulose acetate, enthalpy of mixture. 


\section{Introdução}

Apesar da grande vantagem de ser sintetizada pela natureza, a celulose não é solúvel em solventes orgânicos convencionais e a introdução de grupos menos polares em suas cadeias poliméricas é bastante comum. $\mathrm{O}$ acetato de celulose, caso particular de material empregado nesse trabalho, é um derivado de celulose tradicional, completamente atóxico que é obtido de uma fonte de celulose de grande pureza como polpa de madeira, linter de algodão ou pasta de celulose. Via de regra, a reação emprega ácido acético e anidrido acético utilizando ácido sulfúrico como catalisador. A acetilação pode ser completa ou não, sendo que para o primeiro caso a celulose incorpora três grupos de radical acetato por cada unidade de glicose. $\mathrm{O}$ meio reacional empregado no processo industrial provoca uma acentuada degradação do polímero de modo que apenas 200-300 unidades em média de $(\beta)$ D-glucopiranose estarão presentes na cadeia ${ }^{[1]}$. Após etapa final de hidrólise é comum encontrar produtos contendo de 2-2,5 grupos de acetato por unidade de glicose no polímero.

Nesse trabalho as hidroxilas livres nas cadeias poliméricas do acetato de celulose foram esterificadas utilizando a trietialmina como catalisador e o dianidrido da 3,3',4,4' benzofenonatetracarboxílico (BTDA) como agente de reticulação. A Figura 1 mostra de forma esquemática e ilustrativa a estrutura química dos produtos obtidos após reação. De uma maneira geral, os processos de entrecruzamento e grafitização são utilizados para melhorar as propriedades de polissacarídeos, formando redes poliméricas tridimensionais que incham rapidamente, absorvendo uma grande quantidade de água. Esses processamentos acabam por transformar cadeias poliméricas lineares em géis ou hidrogéis ${ }^{[2]}$. Hidrogéis são redes poliméricas tridimensionais capazes de absorver grandes quantidades de água ou fluidos biológicos, sem dissolver, como resultado de uma reticulação. Grande atenção vem sendo dada a esses materiais devido a potencial aplicação em bioengenharia, indústria alimentícia, biomedicina, processos de separação, purificação de água,

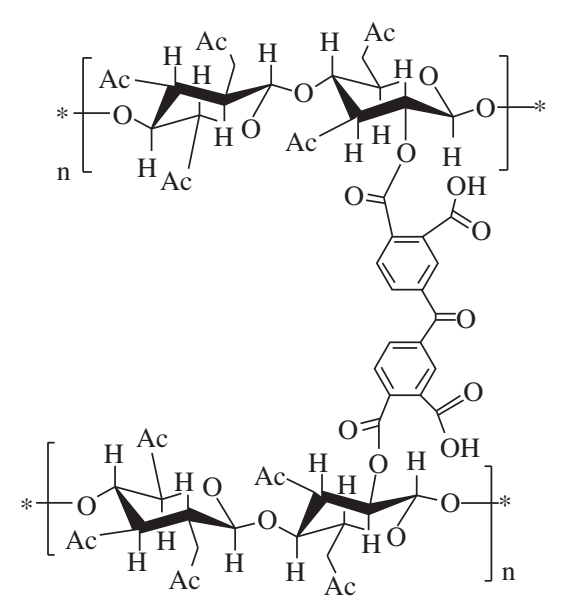

Figura 1. Estrutura química parcial e ilustrativa do hidrogel obtido após a reação de esterificação. cromatografia, agricultura e sistemas de liberação controlada de medicamentos ${ }^{[2-5]}$.

A quantidade de agente reticulador afeta a densidade de reticulação do hidrogel. A insolubilidade da rede e suas complexidades espaciais e topológicas são as principais razões para a dificuldade na sua caracterização ${ }^{[6]}$. De forma adicional, o grau de reticulação na rede polimérica é um fator preponderante para várias propriedades do material, incluindo as térmicas, as mecânicas e a relação entre a estrutura tridimensional da rede e o percentual de inchamento em vários solventes ${ }^{[2]}$.

\section{Experimental}

\section{Materiais}

Acetato de celulose (AC): vendido comercialmente com o nome de Acetol Flakes ${ }^{\circledR}$. Esse material possui grau de substituição (DS) 2,5 e é utilizado como matéria prima para a fabricação de filtros de cigarro. BTDA: fornecido pela Alco Chemical Corporation. O solvente utilizado foi a acetona, fornecida pela Proquímios, com pureza de 99,5\%. O catalisador foi a trietilamina fornecida pela Vetec Química Fina, com pureza de 99\%. Um acetato de celulose padrão fornecido pela ALDRICH, com teor de grupos acetil de $39,8 \%$ p/p e massa molar numérica média (Mn/GPC) de 30,000 foi utilizado como material de referência. Purificação dos reagentes: o acetato de celulose ao ser recebido foi imerso em éter etílico por 30 minutos, em etanol por 1 hora e subsequentemente, lavado com água destilada. As amostras de acetato de celulose purificadas foram submersas em água a $80{ }^{\circ} \mathrm{C}$ por 3 horas e em seguida secas em estufa a vácuo, sob vácuo de $625 \mathrm{~mm} \mathrm{Hg}$, a $80{ }^{\circ} \mathrm{C}$, por 24 horas $^{[7]}$. O BTDA foi colocado em estufa à vácuo, sob vácuo de $625 \mathrm{~mm} \mathrm{Hg}$, a $200{ }^{\circ} \mathrm{C}$, por 2 horas e em seguida guardado em dessecador. A acetona e a trietilamina foram utilizadas como recebidas. $\mathrm{O}$ acetato de celulose padrão e a Avicel foram utilizados como recebidos.

\section{Métodos}

Determinação do ponto de fusão da matéria prima: foi realizada em triplicata, utilizando um aparelho de ponto de fusão da Büchi (Melting point B-540). Análise Térmica Diferencial (DTA): nessa análise, o aparelho utilizado para o estudo foi um METTLER TOLEDO STAR System do laboratório de análises térmicas da UFMG/DQ em condições experimentais variáveis. Determinação do grau de acetilação do Acetol Flakes ${ }^{\circledR}$ : o acetato de celulose foi colocado em estufa sob vácuo de $625 \mathrm{~mm} \mathrm{Hg}$, por 1 hora a $80^{\circ} \mathrm{C}$. Foram pesados em frascos Erlenmeyer exatamente $0,500 \mathrm{~g}$ de acetato de celulose e em seguida adicionou-se $20 \mathrm{~mL}$ de solução etanol/água $75 \% \mathrm{v} / \mathrm{v}$. Os frascos foram tampados e aquecidos por 30 minutos a $60{ }^{\circ} \mathrm{C}$, e em seguida, adicionou-se $25 \mathrm{~mL}$ de solução de $\mathrm{NaOH}(0,5 \mathrm{M})$, previamente padronizada. $\mathrm{O}$ meio reacional foi aquecido, novamente, por 15 minutos, a $60{ }^{\circ} \mathrm{C}$ 
e em seguida deixado para reagir por 72 horas, à temperatura ambiente. $\mathrm{O}$ excesso de $\mathrm{NaOH}$ foi titulado com solução padronizada de $\mathrm{HCl}$ utilizando fenolftaleina como indicador. O DS foi calculado usando a seguinte Equação (1):

$$
\begin{aligned}
& \% \text { Acetyl }=\frac{\left\{\left[\left(\mathrm{V}_{\mathrm{NaOH}} \times \mathrm{A}\right)-\left(\mathrm{V}_{\mathrm{HCl}} \times \mathrm{B}\right)\right]-\left[\left(\mathrm{V}_{\mathrm{NaOH}}^{\prime} \times \mathrm{A}\right)-\left(\mathrm{V}_{\mathrm{HCl}}^{\prime} \times \mathrm{B}\right)\right]\right\} \times 4.3}{\mathrm{w}} \\
& \mathrm{DS}=\frac{3.86 \times(\% \text { Acetyl })}{102.4-(\% \text { Acetyl })}
\end{aligned}
$$

onde \%Acetyl é a porcentagem de gupos acetil na amostra, A é a concentração da solução de $\mathrm{NaOH}$ em mol/L; B é a concentração da solução de $\mathrm{HCl}$ em mol/L; $\mathrm{V}_{\mathrm{NaOH}}$ e V' ${ }_{\mathrm{NaOH}}$ são os volumes da solução de $\mathrm{NaOH}$ adicionados nas amostras e no branco, respectivamente, $\mathrm{V}_{\mathrm{HCl}}$ e $\mathrm{V}^{\prime}{ }_{\mathrm{HCl}}$ são os volumes de $\mathrm{HCl}$ adicionados nas amostras e no branco, respectivamente e w é a massa da amostra em gramas ${ }^{[7]}$. Espectroscopia de absorção no infravermelho (FTIR): o instrumento utilizado para as análises de FTIR foi um Impact 410 (Nicolet). As pastilhas foram preparadas homogeneizando-se 10 partes de $\mathrm{KBr}$ e 1 parte de amostra em um almofariz de ágata. Depois de homogeneizada, a mistura foi prensada e os espectros de transmissão das pastilhas foram então obtidos sem utilização de filtros ou tratamentos matemáticos posteriores. Termogravimetria (TG) e termogravimetria derivada (DTG): todas as medidas foram feitas em atmosfera de nitrogênio com um fluxo de $200 \mathrm{~mL} / \mathrm{min}$, em uma rampa de aquecimento de $10,00{ }^{\circ} \mathrm{C} / \mathrm{min}$ e uma programação de 25,0 a $750,0{ }^{\circ} \mathrm{C}$. $\mathrm{O}$ aparelho utilizado para o estudo foi um METTLER TOLEDO STAR System do laboratório de análises térmicas da UFMG/DQ. Microscopia eletrônica de Varredura (MEV): as análises foram feitas utilizando um microscópio eletrônico de varredura da marca JEOL - 5SM-5510, com imageamento de elétrons secundários e retroespalhados, com sistema EDS/ EBSD acoplados. Voltagem de aceleração $20 \mathrm{Kev}$, spotsze 35, working distance $20 \mathrm{~mm}$ e beam current 50/20 Hz. Adsorção de Nitrogênio (BET) e densidade: A determinação da densidade da amostra foi feita utilizando um Multi pycnometer da QUANTA CHROME à gás (He). A determinação da porosidade dos hidrogéis foi feita utilizando um equipamento da marca Nova 1000 da QUANTA CHROME.

\section{Síntese dos hidrogéis}

$\mathrm{O}$ acetato de celulose foi dissolvido em acetona e meio completamente anidro. Após a dissolução, a solução passou por um processo de filtração para a retirada da fração insolúvel. Após a completa dissolução dos reagentes, o catalisador foi adicionado ao meio reacional, sob agitação constante, e dentro de alguns minutos ocorreu a formação do gel, que pôde ser evidenciada pela brusca mudança na viscosidade do sistema. Hidrogéis de BTDA foram sintetizados com razões estequiométricas de 0,5, 0,75 e 1,0 mol de BTDA/mol de AC fornecendo os produtos 0,$5 ; 0,75$ e 1,0 B, respectivamente. Processo de lavagem e secagem: depois de sintetizado, o gel foi retirado do frasco reacional, titurado manualmente em pedaços de aproximadamente $1 \mathrm{~cm}^{3}$ para facilitar a difusão do excesso de dianidrido para o banho de lavagem e deixado submerso em uma solução etanol/água $20 \%$ v/v, por 10 dias a temperatura ambiente. A partir do décimo dia a solução etanol/água $20 \% \mathrm{v} / \mathrm{v}$ foi substituída por água destilada e o frasco de lavagem foi mantido dentro da estufa à $60{ }^{\circ} \mathrm{C}$ por mais 20 dias. A secagem dos géis foi feita a $50{ }^{\circ} \mathrm{C}$, por 72 horas, em estufa e por 48 horas, a $40^{\circ} \mathrm{C}$, em estufa a vácuo sob vácuo de $625 \mathrm{~mm} \mathrm{Hg}$. Depois de secos os géis foram colocados em um dessecador.

\section{Estudos de inchamento}

Para esses estudos, foram preparadas amostras dos géis em formas cilíndricas com $1,0 \mathrm{~cm}$ de espessura e $2,25 \mathrm{~cm}$ de raio, com volume aproximado de $20 \mathrm{~mL}$. Os géis permaneceram em água com temperatura e tempos variáveis. O percentual de inchamento $(\% \mathrm{~S})$ dos géis em água foi calculado através da seguinte Equação (2):

$$
\% \mathrm{~S}=\left[\left(\mathrm{w}_{\mathrm{t}}-\mathrm{w}_{\mathrm{o}}\right) / \mathrm{w}_{\mathrm{o}}\right] * 100
$$

onde $\mathrm{w}_{\mathrm{t}}$ é a massa de gel inchado no tempo $\mathrm{t}$ e $\mathrm{w}_{\mathrm{o}}$ é a massa de gel seco no tempo 0 .

\section{Discussões}

O ponto de fusão do para o BTDA foi de $204,63{ }^{\circ} \mathrm{C}+/$ $0,028{ }^{\circ} \mathrm{C}$ valor próximo ao encontrado na literatura (Chemy $-Y Q)$ o que reflete a sua relativa pureza.

Os espectros de FTIR dos géis lavados e não lavados são mostrados na Figura 2 e comparados com o espectro do acetato de celulose. As principais diferenças incluem as vibrações angulares fora do plano das ligações $\mathrm{C}-\mathrm{H}$ do anel a $817 \mathrm{~cm}^{-1}$, vibrações de deformação axial do C-O de anidridos cíclicos a $949 \mathrm{~cm}^{-1}$, deformação angular de C-H aromático no plano do anel a $3070 \mathrm{~cm}^{-1}$ e vibração de deformação axial de C-O em ésteres de ácidos aromáticos a $1299 \mathrm{~cm}^{-1}$. A $1730 \mathrm{~cm}^{-1}$ pode ser observado um alargamento e desdobramento da banda de carbonila devido à incorporação de outros ésteres na estrutura, além de carbonila de cetona e ácido carboxílico presentes na estrutura dos agentes de entrecruzamento. Outra

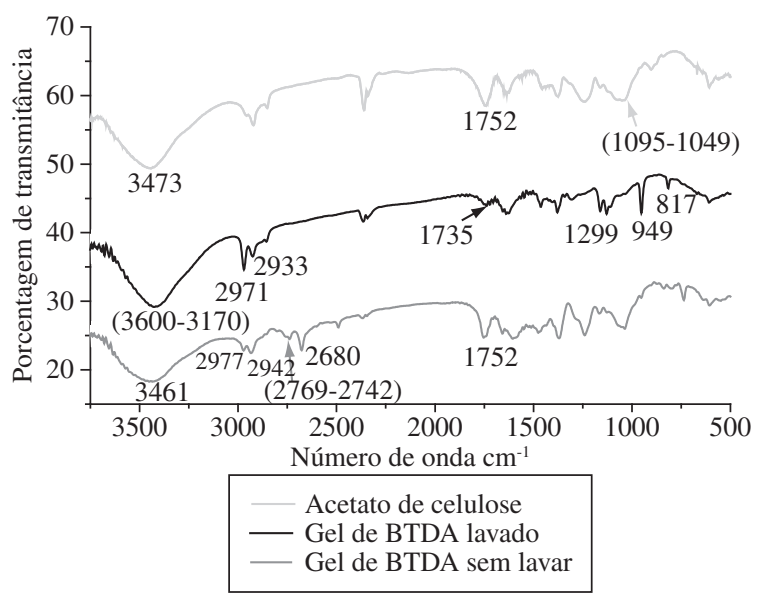

Figura 2. FTIR do gel de BTDA lavado, não lavado e do acetato de celulose. 
importante modificação corresponde ao alargamento da banda a $3480 \mathrm{~cm}^{-1}$ correspondente ao aumento do teor de hidroxilas de grupos carboxílicos após formação da estrutura reticulada. O surgimento de novas bandas é evidência da formação de um novo material e mostra que o produto é composto de uma rede polimérica formada por ligações covalentes, uma vez que, depois de lavados, não houve o completo desaparecimento das bandas de absorção na região aromática dos géis e sim uma diminuição na intensidade dessas bandas.

A Figura 3 mostra as curvas de TG e DTG dos géis obtidos com diferentes razões estequiométricas.

A partir das curvas de TGA e DTG podemos concluir que o entrecruzamento das cadeias de acetato de celulose por qualquer um dos dianidridos leva a uma redução na temperatura de degradação (Td). As curvas DTG mostram dois eventos de perda de massa, o primeiro evento foi atribuído à degradação dos substituintes da cadeia de celulose, isto é, os grupos acetato e os dianidridos e o segundo evento foi

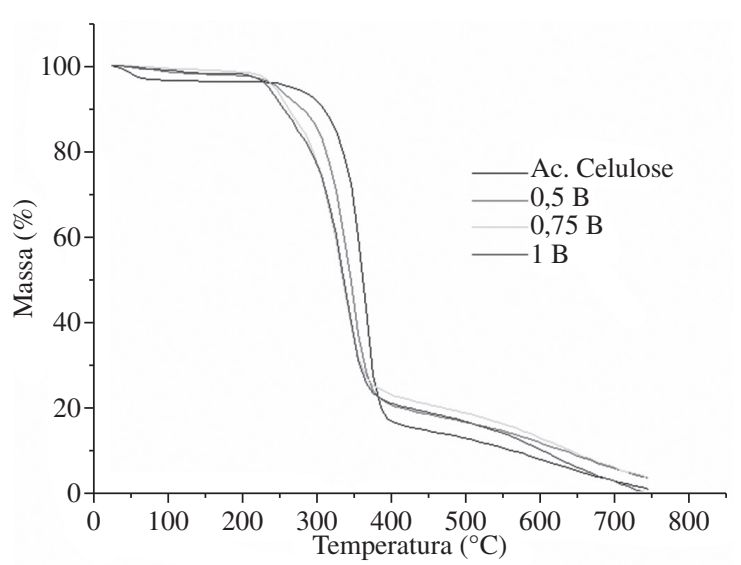

atribuído, principalmente, à degradação da cadeia polimérica do acetato de celulose.

O gel 0,5 B foi analisado por BET e os resultados são apresentados na Tabela 1. Segundo a classificação proposta por Peppas \& Meadows ${ }^{[8]}$ esse hidrogel pode ser classificado como não poroso uma vez que os valores encontrados para os diâmetros médios dos poros foram de $40 \AA$.

As Figuras 4a, b mostram a superfície do Acetol Flakes, e do gel 0,5 B, respectivamente. Os resultados de microscopia eletrônica mostram as modificações superficiais ocorridas após a reação de reticulação e as micrografias mostram que os hidrogéis são não porosos concordando assim com os resultados de BET. As modificações superficiais são evidências da formação de um novo material após a reação de reticulação.

O processo de inchamento de um hidrogel não é descrito unicamente por processos difusionais. A cinética e o mecanismo de inchamento de um hidrogel dependem de

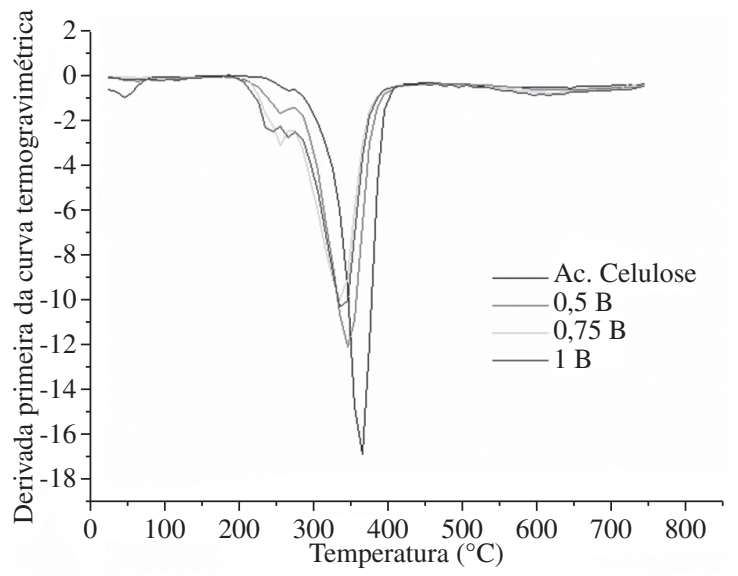

Figura 3. TG e DTG dos géis de BTDA com diferentes razões estequiométricas.

Tabela 1. Porosidade, densidade e superfície específica do gel 0,5 B.

\begin{tabular}{cccccccc}
\hline Gel & $\begin{array}{c}\text { Superfície } \\
\text { especifica }\left(\mathbf{m}^{2} / \mathbf{g}\right)\end{array}$ & $\begin{array}{c}\text { Vol. total dos } \\
\text { poros } \\
\left(\mathbf{c m}^{3} / \mathbf{g}\right)\end{array}$ & $\begin{array}{c}\text { Volume dos } \\
\text { microporos } \\
\left(\mathbf{c m}^{3} / \mathbf{g}\right)\end{array}$ & $\begin{array}{c}\text { Área dos } \\
\text { microporos } \\
\left(\mathbf{m}^{2} / \mathbf{g}\right)\end{array}$ & $\begin{array}{c}\text { Diâmetro médio } \\
\text { dos poros }(\AA)\end{array}$ & $\begin{array}{c}\text { Tamanho máx. } \\
\text { dos poros }(\mathbf{\AA})\end{array}$ & $\begin{array}{c}\text { Densidade } \\
\left(\mathbf{g} / \mathbf{c m}^{3}\right)\end{array}$ \\
\hline $0,5 \mathrm{~B}$ & 2.739 & $2.781 \times 10^{-3}$ & $1.318 \times 10^{-3}$ & 3.732 & $4.062 \times 10^{1}$ & 1437.1 & 1,422 \\
\hline$* \mathrm{R}=0,99996$ (fator de correlação)
\end{tabular}

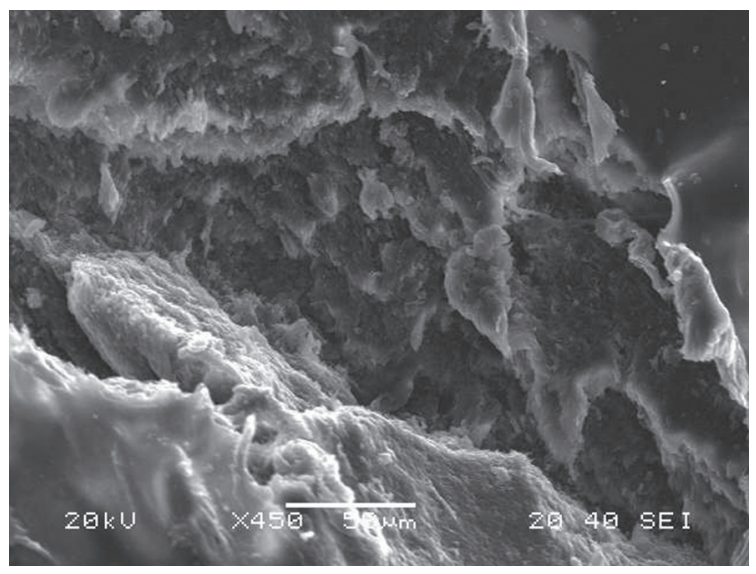

(a)

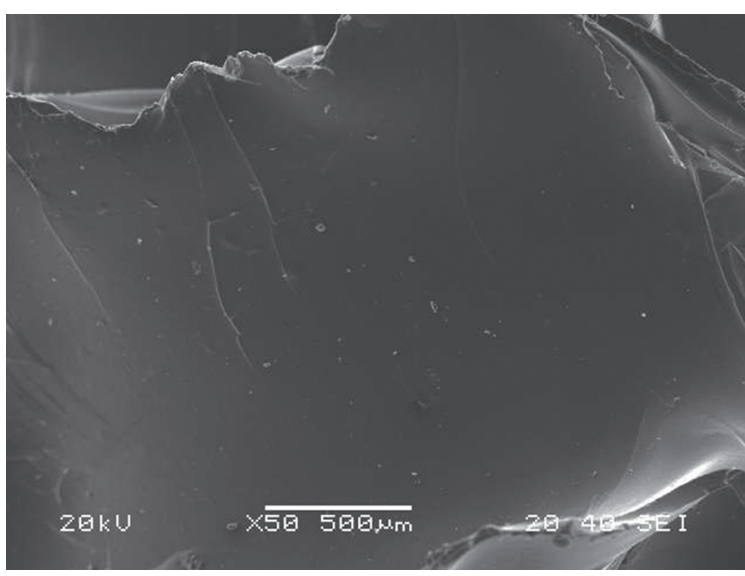

(b)

Figura 4. a) Microfotografia da superfície do AC; e b) da superfície do gel 0.5 de BTDA. 
fatores estruturais como a composição química e a geometria da matriz. Para descrevermos o processo de inchamento adequadamente é importante considerar vários fatores como a geometria exata do hidrogel, o transporte de matéria, a variação no coeficiente de difusão da matriz de acordo com o seu inchamento, o aumento nas dimensões do sistema, a solubilidade das cadeias poliméricas, a presença ou não de poros na matriz e fatores estruturais da rede polimérica como tamanho das cadeias, grau de reticulação, grau de substituição, etc. A dinâmica de inchamento dos hidrogéis é controlada por fatores estruturais da rede polimérica e interações polímero-solvente. Durante a absorção de água a rede polimérica passa de um estado vítreo para um estado borrachoso e a cinética de absorção e transporte de moléculas para dentro do hidrogel é controlado por fatores difusionais dentro de cada uma dessas regiões. É bastante claro que fenômenos de transporte, como a difusão, não ocorrerão do mesmo modo nas duas fases diferentes ${ }^{[9]}$.

As Figuras 5 e 6 mostram as cinéticas de inchamento dos géis à temperaturas do meio controladas e iguais a 20 , 30 e $40{ }^{\circ} \mathrm{C}$, respectivamente. Os gráficos mostram que, de uma maneira geral, um aumento no grau de reticulação leva a um aumento no percentual de inchamento para todos os

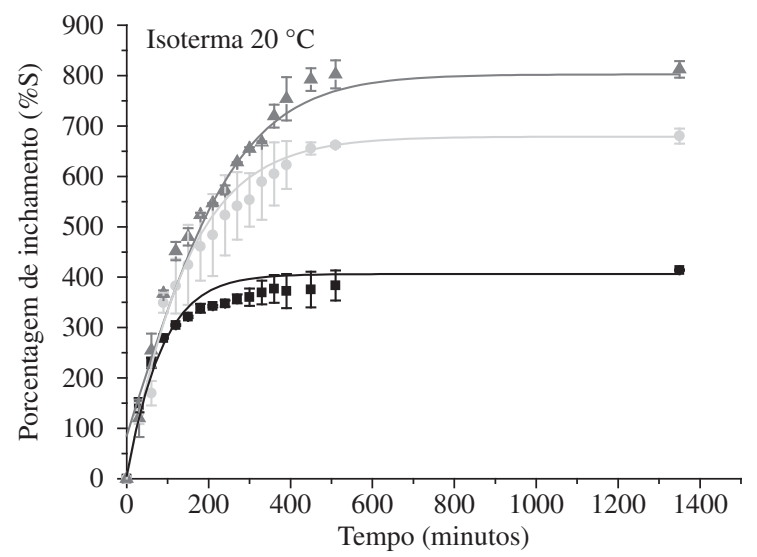

tempos monitorados após imersão. O aumento de absorção de água pelos géis se deve ao aumento na quantidade de grupos hidrofílicos nas cadeias poliméricas de AC devido a inclusão de uma maior extensão de grupos carboxílicos na estrutura do material. Os grupos carboxílicos presentes nas moléculas de dianidrido são capazes de formar ligações de hidrogênio com a água, e dessa forma provocarem o inchamento do gel. Outro fator importante é que em água destilada ( $\mathrm{pH} \cong 6,5)$ os grupos carboxílicos estarão desprotonados e provocam uma repulsão das cadeias poliméricas, ricas em cargas negativas $\left(-\mathrm{COO}^{-}\right)$, assim, facilitam a difusão da água para dentro da matriz polimérica, bem como o relaxamento das cadeias. Nesse caso, a água funciona como plastificante, pois aumenta a mobilidade da rede diminuindo a interação entre as cadeias de acetato de celulose. Esse aumento de mobilidade é um dos motivos que permite com que os hidrogéis sofram grandes aumentos de volume, como mostrado na Figura 5. Pode ser verificado que um aumento na concentração de grupos iônicos (-COO-) na rede polimérica, leva a um aumento no percentual de inchamento do gel, pois a presença desses grupos carregados torna a rede polimérica mais hidrofílica aumentando a afinidade desta pelas moléculas de água.

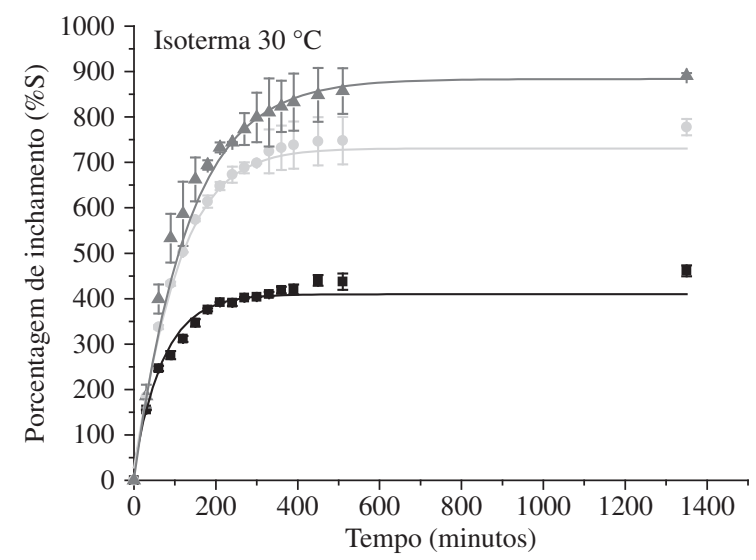

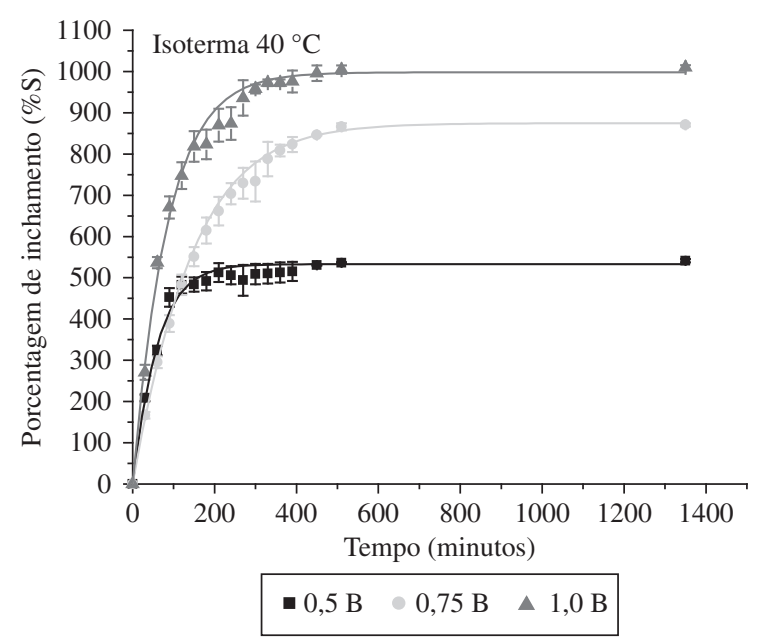

Figura 5. Cinéticas de inchamento para os géis de BTDA com diferentes razões estequiométricas em temperaturas controladas: 20,30 e $40{ }^{\circ} \mathrm{C}$. 
Pode ser verificado que um aumento da temperatura leva igualmente a um aumento no percentual de inchamento do gel, provavelmente devido a um aumento da agitação molecular do meio e afastamento das cadeias em maior extensão (Figura 6).

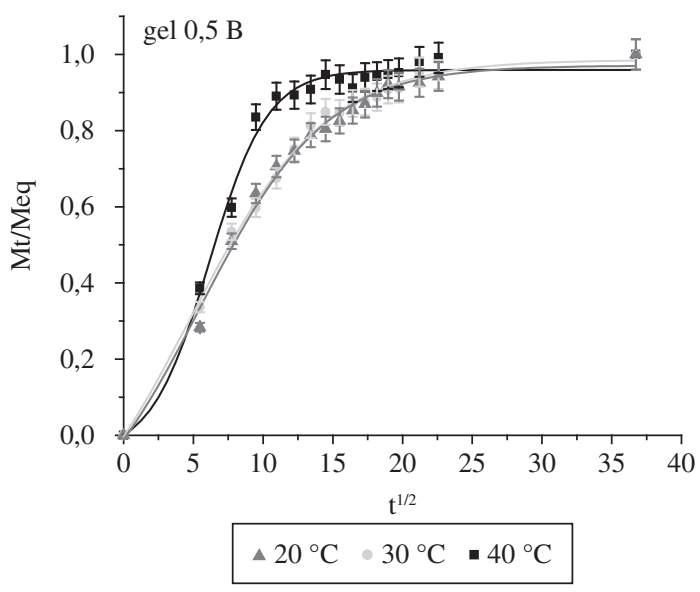

(a)

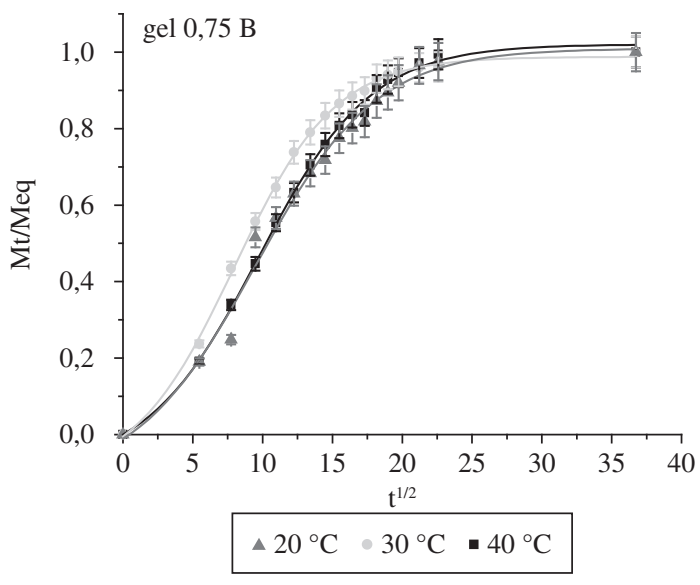

(b)

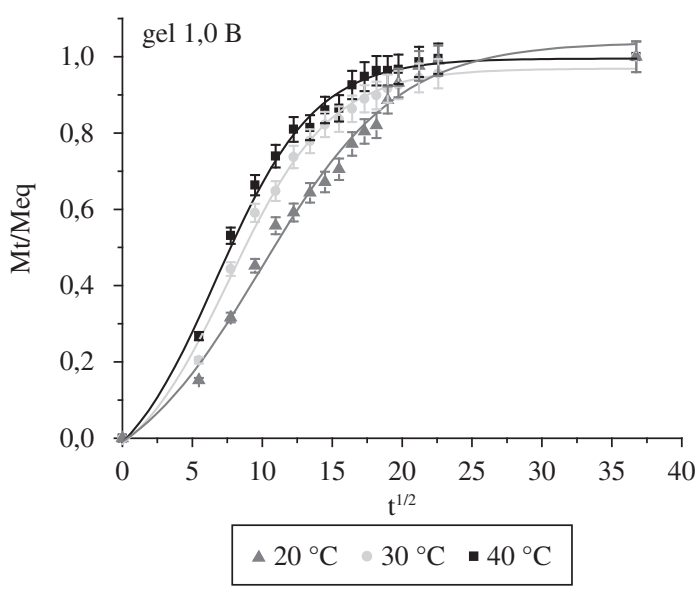

(c)

Figura 6. Mt/Meq em função da raiz quadrada do tempo, a diferentes temperaturas para os diferentes géis: Figura $7 \mathrm{a}-$ gel $0,5 \mathrm{~B}$; Figura $7 \mathrm{~b}$ - gel 0,75; e Figura 7c - gel 1,0 B.
A Figura 6 mostra as curvas obtidas para a fração de água absorvida em função da raiz quadrada do tempo. As curvas de Mt/Meq em função de $\mathrm{t}^{1 / 2}$ mostram que o mecanismo de difusão em géis de BTDA é mais voltado para o tipo II do que para o mecanismo do tipo Fickiano. Uma simples observação das curvas para os valores onde $0,2<\mathrm{Mt} / \mathrm{Meq}<0,6$ para os géis 1,0 e $0,75 \mathrm{~B}$, mostra que o perfil de inchamento pode ser considerado linear entre esses valores. Para o gel 0,5 B (20 e $40{ }^{\circ} \mathrm{C}$ ) não existem pontos suficientes nesse intervalo para comprovar essa informação.

A Tabela 2 mostra os valores encontrados para os coeficientes de difusão dos diferentes géis. Os resultados mostram que, o aumento no grau de reticulação e o aumento na temperatura, provocaram um aumento no coeficiente de difusão dos géis. $\mathrm{O}$ aumento no grau de reticulação provocou um aumento no coeficiente de difusão sendo que esse resultado pode ser explicado com base no aumento do caráter hidrofílico da rede polimérica que facilita a difusão das moléculas de água.

A energia de ativação para o processo de inchamento foi determinada utilizando a equação de Arrhenius:

$$
\mathrm{D}=\mathrm{D}_{\mathrm{o}} \mathrm{e}^{(-\mathrm{ED} / \mathrm{RT})}
$$

onde $\mathrm{E}_{\mathrm{D}}$ é a energia de ativação para o processo de difusão, $\mathrm{R}$ é a constante dos gases e $\mathrm{T}$ é a temperatura em Kelvin. É importante destacar que a linearização da equação de Arrhenius é um tratamento matemático aproximado, mas bastante difundido e aceito na literatura ${ }^{[10]}$, embora melhores resultados possam ser obtidos a partir do uso de técnicas de estimação não-lineares de parâmetros. Os valores para a energia de ativação foram obtidos através da inclinação da reta obtida quando se constrói o gráfico de $\operatorname{lnD}$ em função de $1 / T^{[10]}$. A Tabela 3 mostra as energias de ativação para $o$ processo de difusão para os géis.

Como observado na Tabela 3, um aumento no grau de reticulação provocou um aumento nos valores da $E_{D}$. Novamente, dois fatores estão contribuindo para a energia de ativação do processo de difusão, o caráter hidrofílico da rede e o número de pontos de reticulação.

A entalpia de mistura $\Delta \mathrm{H}_{\text {mix }}$ do sistema gel-água pode ser determinada através da medida da quantidade máxima de água absorvida no equilíbrio a diferentes temperaturas,

Tabela 2. Coeficientes de difusão dos géis de BTDA a diferentes temperaturas.

\begin{tabular}{cccc}
\hline C. Dif. (D) & $\mathbf{0 , 5} \mathbf{B}$ & $\mathbf{0 , 7 5} \mathbf{B}$ & $\mathbf{1 , 0} \mathbf{B}$ \\
\hline $20{ }^{\circ} \mathrm{C}$ & $9,79 \times 10^{-7}$ & $12,23 \times 10^{-7}$ & $10,92 \times 10^{-7}$ \\
$30{ }^{\circ} \mathrm{C}$ & $9,28 \times 10^{-7}$ & $14,80 \times 10^{-7}$ & $29,54 \times 10^{-7}$ \\
$40^{\circ} \mathrm{C}$ & $10,13 \times 10^{-7}$ & $15,55 \times 10^{-7}$ & $32,18 \times 10^{-7}$ \\
\hline
\end{tabular}

Tabela 3. Energias de ativação para o processo de difusão para os géis de BTDA.

\begin{tabular}{cccc}
\hline Gel & $\mathbf{0 , 5} \mathbf{P}$ & $\mathbf{0 , 7 5} \mathbf{P}$ & $\mathbf{1 , 0} \mathbf{P}$ \\
\hline$\Delta \mathrm{E}_{\mathrm{D}}(\mathrm{kJ} / \mathrm{mol})$ & $*$ & 9,19 & 41,54 \\
\hline
\end{tabular}




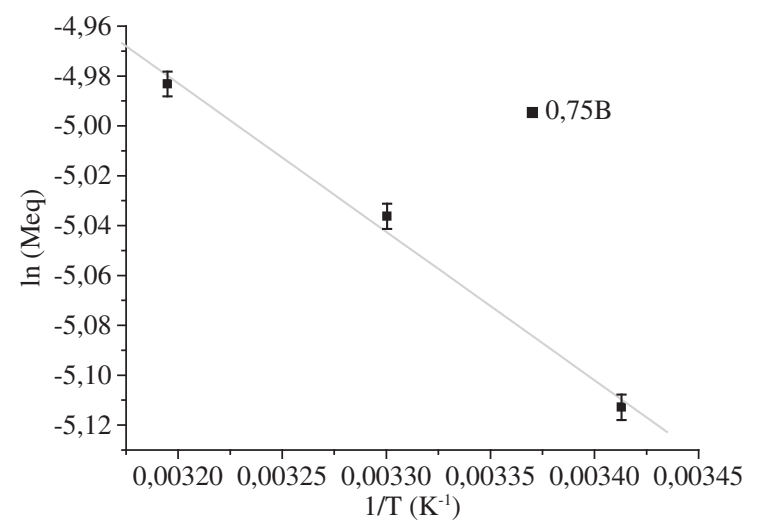

Figura 7. Gráfico de $\ln \mathrm{M}_{\mathrm{eq}}$ em função de $1 / \mathrm{T}$ para o gel $0,75 \mathrm{~B}$ empregado como exemplo para esse estudo.

usando a equação de Gibbs/Helmholtz aplicada ao gel 0,75 B (Figura 7):

$$
\mathrm{dln}_{\mathrm{eq}} / \mathrm{d}(1 / \mathrm{T})=-\Delta \mathrm{H}_{\text {mix }} / \mathrm{R}
$$

onde $\mathrm{R}$ é a constante dos gases e $\mathrm{T}$ é a temperatura absoluta ${ }^{[10]}$. O gel 0,75B mostrou uma entalpia de mistura positiva com valor de $4,92 \mathrm{~kJ} / \mathrm{mol}$ e portanto tratando-se de um processo endotérmico. Esse resultado nos diz que o processo de relaxação das cadeias poliméricas deve ser ativado para que o processo de inchamento ocorra assim como para os géis de PMDA.

\section{Conclusões}

Hidrogéis de acetato de celulose com grau de substituição 2,5 foram sintetizados utilizando BTDA como agente entrecruzante. Os hidrogéis foram classificados como não porosos uma vez que apresentaram diâmetro médio dos poros de 40,62 A. As isotermas de inchamento para os dois hidrogeis mostraram que um aumento no grau de reticulação provocou um aumento no percentual de água absorvida no equilíbrio (\%Seq) o que foi atribuído ao aumento no caráter hidrofílico da rede. O \%Seq também aumentou com o aumento da temperatura o que foi atribuído ao aumento na energia térmica das moléculas de água, aumento na mobilidade das cadeias poliméricas e no aumento na quantidade de grupos iônicos dentro da rede. A análise do mecanismo de absorção de água pelos hidrogéis, para os primeiros $60 \%$ das isotermas, se mostrou anômalo, onde concluímos que presença de grupos iônicos influencia o mecanismo do tipo Fickiano, pois a presença de cargas na rede aumenta a afinidade entre a rede e as moléculas de água, provocando a difusão das moléculas de água para o interior da rede. Por outro lado, os mesmos grupos iônicos responsáveis pela ativação do mecanismo do tipo Fickiano, por estarem todos carregados negativamente, provocam uma repulsão das cadeias poliméricas favorecendo o mecanismo do tipo II. Dada a grande capacidade de absorção de água, a baixa demanda energética, o baixo custo das matérias primas utilizadas na preparação dos géis, a possibilidade de obtermos hidrogéis com razões estequiométricas muito baixas, a utilização de matéria prima renovável e a simplicidade do processo de síntese desses hidrogéis. Hidrogéis de acetato de celulose reticulados com BTDA podem ser um interessante material para o uso em sistemas de liberação controlada de medicamentos, processos de tratamento de água e processos de separação e purificação.

\section{Referências Bibliográficas}

1. Oliveira Júnior, A. R. - "Obtenção e caracterização de acetato de celulose modificado com organossilano", Tese de Doutorado, Universidade Estadual de Campinas, Brasil (2002).

2. Singh, B.; Chauhan, G. S. S. \& Nirmala Chauhan, K. Carbohydrate Polymers, 67, p.190 (2002).

3. El-hag Ali, A.; Shawky, H. A.; Abd El Rehim, H. A. \& Hegazy, E. A. - European Polymer Journal, 39, p.2337 (2003).

4. Karadag, E.; Saraydin, D. \& Güven, O. - NIMB Beam Interactions with Materials \& Atoms, 225, p.489 (2004).

5. Jabbari, E. \& Nozari, S. - European Polymer Journal, 36, p.685 (2000).

6. Kioussis, D. R. \& Kofinas, P. - Polymer, 46, p.10167 (2005).

7. Gocho, H.; Shimizu, H.; Tanioka, A.; Chou, T. J. \& Nakajima, T. - Carbohydrate Polymers, 41, p.83-86 (2000).

8. Ende, M. T. \& Peppas, N. A. - Journal of controlled release, 48, p.47 (1997).

9. Peppas, N. A. \& Khare, A. R. - Advanced Drug Dellivery Reviews, 11, p.1 (1993).

10. Peniche, C.; Cohen, M. E.; Vázquez, B. \& San Román, J. - Polymer, 38, p.5977 (1997).

Enviado: 31/03/09

Reenviado: 11/08/09

Aceito: 13/08/09 